\title{
Getting More from the Sleep Recording
}

\author{
Walter T. McNicholas, MD, FERS
}

\section{KEYWORDS}

- Obstructive sleep apnea $\bullet$ Signal processing • Oximetry $\bullet$ Acoustic recording $\bullet$ Pulse transit time

- Actigraphy • Biosensors

\section{KEY POINTS}

- Developments in signal processing facilitate the automated analysis of traditional signals such as oxygen saturation and electroencephalogram, which provides superior insight into physiology and pathophysiology compared to manual analysis.

- These developments have resulted in increasing recognition that the traditional measure of sleepdisordered breathing, the apnea-hypopnea index, is a poor predictor of disease significance.

- New approaches to the recording and analysis of traditional signals such as snoring and oxygen saturation facilitate ambulatory diagnosis of obstructive sleep apnea.

- Detailed insight into the characteristics of oxygen desaturation during sleep provides superior prediction of comorbidities than the apnea-hypopnea index.

- Derivatives of the electrocardiogram and pulse wave provide indirect data on sleep-disordered breathing that are suitable for ambulatory diagnosis.

\section{INTRODUCTION}

Sleep recordings have been a feature of clinical sleep practice for more than 4 decades, and full sleep laboratory recordings in the form of polysomnography (PSG) remain largely based on principles established in these early years. Sleep staging remains fundamentally based on the scoring rules established by Rechtschaffen and Kales in 1968, ${ }^{1}$ and the scoring of sleepdisordered breathing (SDB) events is based on the so-called Chicago Criteria introduced in 1999, which also proposed a severity grading for obstructive sleep apnea (OSA) based on the frequency of apneas and hypopneas per hour of sleep (AHI). ${ }^{2}$ However, developments in technology and signal analysis in more recent years offer considerable scope to expand and enhance the information that can be obtained from sleep studies, and advances in the understanding of mechanisms contributing to cardiometabolic comorbidities offer new insights regarding the most important sleep-related variables that contribute to these comorbidities. ${ }^{3}$ There has been increasing interest among the sleep research community in exploring new and novel approaches to the diagnosis of sleep disorders, especially OSA, and there is increasing recognition that variables such as the AHI may not be the most important measure to quantify the severity of the disorder. ${ }^{4}$

The focus of this review is to explore how additional information may be obtained from signals obtained in traditional sleep recordings such as laboratory-based PSG and home-based sleep studies, as well as information from additional signals that do not form part of traditional sleep studies. The review will focus primarily on the assessment of patients with suspected OSA, as the high prevalence of this disorder, which affects up to one billion subjects worldwide, ${ }^{5}$ makes it particularly relevant to explore novel approaches to the accurate and reliable diagnosis of this disorder in the ambulatory setting. ${ }^{6}$

Currently, disease severity is measured using $\mathrm{AHI}$ as determined from a sleep study. However, there is poor association between daytime

Department of Respiratory and Sleep Medicine, School of Medicine, University College Dublin, St. Vincent's Hospital Group, Elm Park, Dublin 4, Ireland

E-mail address: walter.mcnicholas@ucd.ie 
symptoms such as excessive daytime sleepiness and the $\mathrm{AHI},{ }^{7}$ and there is increasing evidence that cardiometabolic comorbidities may be more related to measures of oxygen desaturation during sleep than the AHI. ${ }^{3}$ Thus, there is increasing interest in moving away from the $\mathrm{AHI}$ as the most important measure of OSA severity toward a more personalized approach to OSA diagnosis and treatment, ${ }^{8}$ which considers individual risk factors, clinical history, and comorbid disease in the diagnosis and treatment of each patient with OSA.

\section{INDIVIDUAL PATIENT PHENOTYPING}

While overnight diagnostic sleep studies provide the core evidence of SDB in patients with suspected OSA, there is clear evidence that the sleep study alone does not provide sufficient evidence for the diagnosis of the clinical syndrome. This aspect has been recognized for many years, ${ }^{9,10}$ and more recently, there is strong evidence that clusters of different clinical phenotypes can be identified among the broad population of patients presenting for assessment. ${ }^{11}$ Furthermore, certain pathophysiological traits that are very common in OSA such as loss of nocturnal dipping of blood pressure (BP) have significant implications for the development of associated comorbidity. ${ }^{12}$ Thus, whatever sleep study is used in the assessment of suspected OSA, the findings must be integrated into the overall assessment of the patient as regards clinical significance, and management should be linked to the underlying clinical and pathophysiological phenotypes where additional factors to the AHI such as acute systemic effects and associated relevant comorbidity are factored into the decision-making process. ${ }^{8}$ A further consideration in the clinical assessment of OSA is the role of the $\mathrm{AHI}$ alone, referred to here in this context as SDB. Although the International Classification of Sleep Disorders refers to $\mathrm{AHI} \geq$ 15 as sufficient for a diagnosis of OSA, this appears questionable in the context of this level of SDB being reported in up to $50 \%$ of a normal adult male population. ${ }^{13}$

\section{DEVELOPMENTS IN THE ANALYSIS OF EXISTING SIGNALS}

Core signals relevant to sleep and breathing disorders such as airflow, oxygen saturation, and cardiac variables have been included in sleep studies for decades, but developments in signal analysis have permitted enhanced and clinically relevant information to be obtained, which significantly adds to the diagnostic potential of the studies concerned. Furthermore, novel approaches to the analysis of these traditional signals may facilitate the use of limited diagnostic systems that may be especially useful in the ambulatory setting.

\section{ELECTROENCEPHALOGRAPHY AND SLEEP STAGING}

Developments in electroencephalographic (EEG) recording technology have permitted the introduction of ambulatory EEG recordings, which may use a full EEG montage. This technology is not necessary in most clinical situations involved in the assessment of OSA, although some limited ear-based EEG recording systems have been developed that are easy to apply and may provide additional useful information in the ambulatory respiratory-sleep clinical setting. ${ }^{14}$

Specialized computer-based analysis of the EEG by techniques such as spectral analysis provides additional insight into sleep physiology and pathology beyond traditional sleep staging, but these are research-oriented and have little application in the clinical setting of respiratory sleep disorders. ${ }^{15,16}$

\section{ACOUSTIC AND AIRFLOW DEVICES Acoustic Devices}

Although snoring is a common feature in patients with OSA, the symptom has limited value on its own in the assessment of OSA because of its weak relationship with the AHI. ${ }^{17}$ Nonetheless, the detailed characteristics of snoring and, especially, the characteristic intermittent nature of snoring in patients with OSA provide potential diagnostic utility both alone and in combination with other signals. In this context, periods of apnea/hypopnea have quite different acoustic characteristics to nonapneic snoring. One such acoustic device is BresoDX (BresoTEC Inc, Toronto, Ontario, Canada), which is a portable device that consists of a lightweight face frame, which contains an embedded electronic module and microphone. Recorded sounds are continually stored and can subsequently be downloaded for analysis. ${ }^{18,19}$ As might be expected, a characteristic cyclical intermittent pattern of snoring has the greatest predictive potential for the diagnosis of OSA. In one report of 135 subjects with suspected OSA, the calculated AHI using BresoDX showed a relatively good correlation with PSG and demonstrated a diagnostic accuracy ranging between $88.9 \%$ and $93.3 \%$ at $\mathrm{AHI}$ cutoffs of 5 to $15 .^{20}$ More recently, an over the counter small, wireless wearable patch has been developed 
(Zansors, Arlington, VA), which estimates breathing patterns using an inbuilt microphone and includes an accelerometer to record movement. A pilot study of the device demonstrated $75 \%$ sensitivity and $71 \%$ specificity for detecting SDB compared to gold standard PSG. ${ }^{21}$

\section{Airflow Devices}

Devices that record airflow by nasal pressure recordings as a single measure have been developed as a more simple ambulatory diagnostic technique for OSA, and an example of such a device is the ApneaLink (Resmed, Sydney, Australia). ${ }^{22}$ One report comparing the device with PSG showed a $73.1 \%$ sensitivity and $91 \%$ specificity for detecting an AHI greater than 15 in at-risk populations, ${ }^{23}$ with similar results reported in other studies. ${ }^{22,24}$

\section{OXIMETRY}

Overnight oximetry has long been proposed as a simple and reasonably accurate technique for OSA diagnosis, especially in severe cases, ${ }^{10,25}$ but has limited reliability in mild OSA where oxygen desaturations may be relatively minor. To be useful in the assessment of patients with OSA, arterial oxygen saturation $(\mathrm{SpO} 2)$ recordings require a high sampling rate $(>0.5 \mathrm{~Hz})$ to ensure detection of the intermittent oxygen desaturations that are characteristic of the disorder. In clinical practice, several relevant variables may be obtained from the recordings, including the oxygen desaturation index (ODI), which is the number of desaturations per hour which drop $\geq 3 \%$ (ODI3) or $\geq 4 \%$ (ODI4) below the baseline level, and the cumulative time with an $\mathrm{SpO} 2$ below a predetermined level, usually $90 \%$ (T90). Additional information can be obtained from the SpO2 variability, referred to as the delta index. ${ }^{26,27}$ In OSA, a "saw-tooth" pattern of recurring transient oxygen desaturations is seen, especially in severe cases, which provides a unique visual picture of the disorder. Simple indices such as the ODI may fail to capture all the important and potentially relevant pathophysiological characteristics ${ }^{25}$ and novel strategies for the analysis of oximetry using automated techniques ${ }^{28-30}$ to help maximize the diagnostic potential of $\mathrm{SpO} 2$ data. Furthermore, novel approaches to oxygen desaturation such as the hypoxic burden have been demonstrated to provide a superior prediction of cardiovascular morbidity and mortality than the $\mathrm{AHI}$ in large-population studies. ${ }^{31}$

Computer-assisted applications that provide automated signal processing facilitate the quantification of the frequency, duration, and severity of desaturations, which enhance the clinical assessment of OSA. ${ }^{29,32-34}$ Some devices use smartphone-based technology or wearable applications as the receiver which may have potential for home screening to prioritize cases for more detailed investigation ${ }^{35-37}$ and may also have a role in treatment follow-up. ${ }^{38}$

\section{Oximetry and Apneas and Hypopneas per Hour of Sleep}

The ODI may be used as an alternative to the $\mathrm{AHI}$ to quantify the number of respiratory events during the night but may underestimate the severity of OSA with potentially important clinical consequences ${ }^{39,40}$ that may influence treatment decisions. ${ }^{26}$ However, a more detailed analysis of the $\mathrm{SpO} 2$ signal with computer-assisted and machine learning algorithms improves the predictive ability ${ }^{41-43}$ with a diagnostic accuracy of up to $96.7 \%$ reported. Furthermore, there is growing evidence that measures of oxygen desaturation are superior to $\mathrm{AHI}$ in the prediction of comorbidities including hypertension (HTN), ${ }^{44}$ diabetes mellitus, ${ }^{45}$ and heart failure. ${ }^{46}$

Oximetry may also have value in screening patients for OSA in certain comorbidities including stroke, ${ }^{47}$ heart failure, ${ }^{48}$ morbid obesity, ${ }^{49,50}$ and chronic obstructive pulmonary disease (COPD). ${ }^{51}$ In stroke, OSA is associated with diminished recovery and increased mortality. ${ }^{52}$ In patients with congestive cardiac failure, overnight oximetry has a high sensitivity $(97 \%)$ but poor specificity $(32 \%)$ for SDB. ${ }^{48}$ Oximetry performs better in patients with gross obesity with reports of up to $100 \%$ sensitivity and $93 \%$ specificity for SDB in ambulatory testing. ${ }^{49}$ Oximetry has limited potential for the diagnosis of OSA in patients with COPD with low sensitivity (59\%) and specificity $(60 \%)^{51}$ although performs better when combined with novel signal analysis technology. ${ }^{53}$

\section{Cardiac Based Measures}

Electrocardiograph (ECG) analysis: monitoring of heart rate variability (HRV), electrocardiograph morphology, and respiration

Monitoring of HRV by overnight ECG has long been recognized as a potential diagnostic tool in patients with SDB. ${ }^{54}$ In standard PSG, a singlelead ECG is recorded to allow measurement of heart rate and rhythm. A special dedicated software program allows analysis of $\mathrm{HRV}^{55}$ that can provide information relating to autonomic activity and may give added insight into sleep stages. ${ }^{56}$ Further information can be obtained from fluctuations in the QRS amplitude that are a consequence of rib cage movements during respiration. The 
combination of ECG-derived respiratory movement and sleep apnea-related HRV has the potential to be a useful screening tool for $\mathrm{OSA}^{57}$ and has the added value of being available in a typical cardiology setting, which provides the potential for screening in this setting before referral to a sleep clinic.

\section{Pulse transit time}

The pulse transit time (PTT) is a variable that is derived from the ECG and the arterial pressure wave measured by a finger probe and has been reported to reflect inspiratory effort. ${ }^{58} \mathrm{PTT}$ measures the time taken for the arterial pulse wave to travel between the aortic valve (R wave on ECG) and the finger blood vessels as indicated by pulse oximetry. Pulse wave speed varies with arterial stiffness, which in turn is influenced by the BP level. PTT has been reported to give an indirect measure of both apnea and arousal. ${ }^{58}$

\section{Peripheral arterial tonometry (the pulse wave as a measure)}

Peripheral arterial tonometry (PAT) provides a detailed assessment of the pulse wave and is reported to be a relatively robust diagnostic screening method for OSA. The pulse wave amplitude is influenced by sympathetic tone, and arousals are also associated with a drop in pulse amplitude. Furthermore, the pulse rate also provides an indirect assessment of sleep stage. ${ }^{59}$ WatchPAT (Itamar Medical, Caesarea, Israel) uses a proprietary algorithm combining PAT data, oxygen saturation, pulse rate, and actigraphy that generates an estimate of total sleep time and calculates an AHI. ${ }^{60}$ WatchPAT has been evaluated in several studies with some, but not all, reports indicating the device to provide a reasonably accurate assessment of OSA. ${ }^{59-61} \mathrm{~A}$ meta-analysis found a good correlation between sleep indices calculated by laboratory-based PSG and those obtained from a PAT device $(r=0.889),{ }^{62}$ thus supporting its use as a viable ambulatory diagnostic tool for OSA.

\section{Blood pressure monitoring as a potential diagnostic tool}

OSA is a recognized independent risk factor for systemic HTN, and obstructive apneas may lead to acute BP elevation during sleep, ${ }^{12,63}$ which can result in a loss of the normal nocturnal dipping pattern of BP. A recent meta-analysis found that OSA is associated with a 1.5-fold increase in the prevalence of nondipping $\mathrm{BP},{ }^{64}$ thus suggesting that ambulatory BP monitoring (ABPM) could serve as a surrogate marker for OSA. Support for this possibility comes from reports that nondipping nocturnal BP predicts OSA in subjects undergoing
ABPM. ${ }^{65}$ Furthermore, a recent report from this department indicated a high predictive value for moderate to severe OSA in unselected patients recruited from a HTN clinic who had a nondipping pattern of nocturnal BP on 24-hour ABPM. ${ }^{66}$ These findings support the possibility that ABPM may be a useful biomarker for OSA, irrespective of the clinical index of suspicion for the disorder.

Limitations of ABPM with a pneumatic cuff include the possibility that cuff inflation causes arousal and that the device is unable to track rapid changes in BP associated with individual apneas. ${ }^{67,68}$ Such limitations may be overcome by continuous measurement of BP by finger photoplethysmography, which uses a small cuff fitted to the finger that provides a continuous measurement. This device provides beat-by-beat pressure measurements and gives an indication of BP variability, which is typically increased in OSA. ${ }^{69} \mathrm{~A}$ novel smart-watch, CareUp (Farasha Labs, Paris, France), has been developed that gives a continuous estimation of $\mathrm{BP}^{70}$ and has been validated in a study of 44 subjects. ${ }^{67}$ This simple device may provide an additional method for home BP monitoring, which could be a promising screening method for OSA, although further research of this technique is required before being accepted into clinical practice.

\section{ACTIGRAPHY}

Sleep is associated with reduced body motion compared to wakefulness, and lack of body movement is widely used as a surrogate marker for sleep. Actigraphy is the most widely used method, and typically uses an accelerometer that is either a stand-alone device or built into a wristwatch. Actigraphy can be used to estimate daily sleepwake cycles, which can be clinically useful in the evaluation of many sleep disorders. Actigraphy has been formally evaluated by the American Academy of Sleep Medicine and recognized as a valid research tool in sleep ${ }^{71}$ and, more recently, as an alternative to PSG for prolonged monitoring of sleep quality. ${ }^{72}$ Several reviews ${ }^{73-75}$ have indicated that actigraphy can provide clinically useful information about sleep in the natural environment, which may assist in clinical decisionmaking. However, actigraphy is not reliable in distinguishing different sleep stages and has poor specificity. ${ }^{76}$ In comparison with PSG, actigraphy overestimates total sleep time and underestimates time spent awake. ${ }^{77}$ Actigraphy provides an indirect signal relating to body motion, which implies that estimation of sleep stages is only derived from an assessment of how body motion changes during different sleep stages, and is likely 


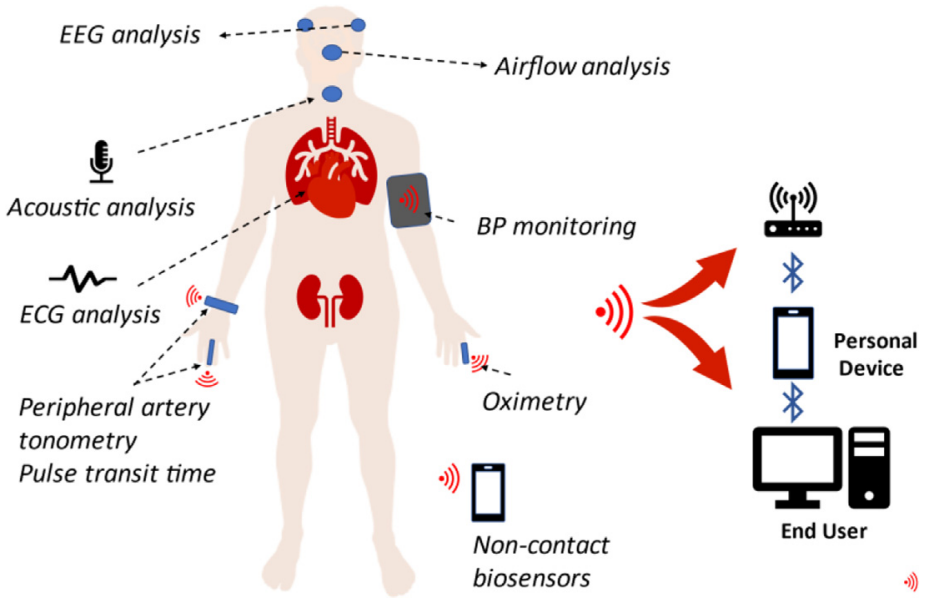

Fig. 1. Potential physiologic signals for the diagnosis and monitoring of OSA. Signals can be fed wirelessly or via Bluetooth to a router or smartphone and then uploaded to a secured database, whereby end users may access and review the data. BP, blood pressure; ECG, electrocardiogram; EEG, electroencephalogram. to be of limited accuracy in this determination. However, with improving technology, the role of actigraphy as a screening tool will likely increase and be included in ambulatory diagnostic systems for OSA where accurate measures of sleep staging are not generally required.

\section{Wireless Systems - Biosensors in Obstructive Sleep Apnea}

Wireless monitoring systems have been in clinical use for several decades, and an early example involved pressure-sensitive foils placed over the mattress, which provided measures of sleep, heart rate, and respiration. ${ }^{78}$ Wireless devices have greatly improved over subsequent years and benefit from developments in digital technology that are used in signal acquisition and processing.

Radio frequency waves, which are similar to radar technology, can detect small body movements, such as those produced by respiration, which may facilitate the assessment of sleep and wakefulness and of SDB. SleepMinder (ResMed Sensor Technologies, Dublin, Ireland) is a noncontact device that estimates the severity of SDB using a multichannel biomotion sensor and an integrated analysis software program. ${ }^{79}$ The device has been shown to correlate well with PSG in the determination of $\mathrm{AHI}^{80}$ and sleep efficiency ${ }^{81}$ during controlled laboratory settings. More recently, the device has been reported to be a useful screening tool in the detection of moderate and severe OSA, although having poor accuracy in the setting of mild OSA. ${ }^{82}$

Actigraphy has been incorporated into many smartphone applications that estimate sleep quality, and additional signals relevant to the assessment of SDB may be obtained from audio and oximetry recordings that have been incorporated into many wearable recording systems, which may also include monitoring of heart rate. ${ }^{83}$ Given their wide availability, relatively low cost, and ease of use, smartphones and wearable devices may provide a valuable screening opportunity for the detection of OSA and other sleep disorders, ${ }^{84,85}$ which may also help prioritize patients that require more detailed investigation. Finally, technological advances in telemedicine may strengthen interdepartmental collaboration to improve the overall care of OSA patients. ${ }^{86}$

\section{SUMMARY}

Developments in signal technology and analysis provide novel approaches to the assessment of patients suspected of OSA, which range from enhanced analysis of traditional signals to novel signal technologies that provide surrogate markers of OSA. The potential range of novel approaches to the assessment of OSA is illustrated in Fig. 1.

\section{CLINICS CARE POINTS}

- When examining the oximetry tracing for features consistent with OSA, ensure that the technology of the recording device has a sufficiently high sampling rate to detect the characteristic fluctuations in oxygen saturation.

- While snoring does not directly relate to OSA, short gaps during prolonged periods of snoring provide an indirect indication of upper airway obstruction. 
- The absence of nocturnal dipping of blood pressure is a potential surrogate marker of OSA and is especially important as this represents a cardiovascular comorbidity.

\section{DISCLOSURE}

The author has nothing to disclose.

\section{REFERENCES}

1. Rechtschaffen A, Kales A. A manual of standardized terminology, techniques and scoring system for sleep stages of human subjects. Washington, DC: US Government Printing Office, Public Health Service; 1968.

2. Flemons WW, Buysse D, Redline S, et al. Sleeprelated breathing disorders in adults: recommendations for syndrome definition and measurement techniques in clinical research. Sleep 1999;22(5): 667-89.

3. McNicholas WT. Obstructive sleep apnoea and comorbidity - an overview of the association and impact of continuous positive airway pressure therapy. Expert Rev Respir Med 2019;13(3):251-61.

4. Pevernagie DA, Gnidovec-Strazisar B, Grote L, et al. On the rise and fall of the apnea-hypopnea index: a historical review and critical appraisal. J Sleep Res 2020;29(4):e13066.

5. Benjafield AV, Ayas NT, Eastwood PR, et al. Estimation of the global prevalence and burden of obstructive sleep apnoea: a literature-based analysis. Lancet Respir Med 2019;7(8):687-98.

6. O'Mahony AM, Garvey JF, McNicholas WT. Technologic advances in the assessment and management of obstructive sleep apnoea beyond the apnoeahypopnoea index: a narrative review. J Thorac Dis 2020;12(9):5020-38.

7. Deegan PC, McNicholas WT. Predictive value of clinical features for the obstructive sleep apnoea syndrome. Eur Respir J 1996;9(1):117-24.

8. Randerath W, Bassetti CL, Bonsignore MR, et al. Challenges and perspectives in obstructive sleep apnoea: report by an ad hoc working group of the sleep disordered breathing group of the European respiratory society and the European sleep research society. Eur Respir J 2018;52(3):1702616.

9. Young T, Palta M, Dempsey J, et al. The occurrence of sleep-disordered breathing among middle-aged adults. N Engl J Med 1993;328(17):1230-5.

10. McNicholas WT. Diagnosis of obstructive sleep apnea in adults. Proc Am Thorac Soc 2008;5(2): 154-60.

11. Bailly S, Grote L, Hedner J, et al. Clusters of sleep apnoea phenotypes: a large pan-European study from the European Sleep Apnoea Database (ESADA). Respirology 2021;26(4):378-87.

12. Crinion SJ, Ryan S, McNicholas WT. Obstructive sleep apnoea as a cause of nocturnal nondipping blood pressure: recent evidence regarding clinical importance and underlying mechanisms. Eur Respir J 2017;49(1):1601818.

13. Heinzer R, Vat S, Marques-Vidal P, et al. Prevalence of sleep-disordered breathing in the general population: the HypnoLaus study. Lancet Respir Med 2015; 3(4):310-8.

14. Bleichner MG, Debener S. Concealed, unobtrusive ear-centered EEG acquisition: cEEGrids for transparent EEG. Front Hum Neurosci 2017;11:163.

15. Liu S, Shen J, Li Y, et al. EEG power spectral analysis of abnormal cortical activations during REM/ NREM sleep in obstructive sleep apnea. Front Neurol 2021;12:643855.

16. Mainieri G, Maranci J-B, Champetier P, et al. Are sleep paralysis and false awakenings different from REM sleep and from lucid REM sleep? A spectral EEG analysis. J Clin Sleep Med 2021;17(4): 719-27.

17. Alshaer H, Hummel R, Mendelson M, et al. Objective relationship between sleep apnea and frequency of snoring assessed by machine learning. J Clin Sleep Med 2019;15(3):463-70.

18. Alshaer $H$, Fernie GR, Maki E, et al. Validation of an automated algorithm for detecting apneas and hypopneas by acoustic analysis of breath sounds. Sleep Med 2013;14(6):562-71.

19. Alshaer H, Levchenko A, Bradley TD, et al. A system for portable sleep apnea diagnosis using an embedded data capturing module. J Clin Monit Comput 2013;27(3):303-11.

20. Alshaer H, Fernie GR, Tseng WH, et al. Comparison of in-laboratory and home diagnosis of sleep apnea using a cordless portable acoustic device. Sleep Med 2016;22:91-6.

21. Abbasi J. In-home, over-the-counter sleep apnea sensor on the Horizon. JAMA 2017;317(22):2271.

22. Erman MK, Stewart D, Einhorn D, et al. Validation of the ApneaLink for the screening of sleep apnea: a novel and simple single-channel recording device. J Clin Sleep Med 2007;3(4):387-92.

23. Masa JF, Duran-Cantolla J, Capote F, et al. Effectiveness of home single-channel nasal pressure for sleep apnea diagnosis. Sleep 2014;37(12):1953-61.

24. Oktay B, Rice TB, Atwood CW Jr, et al. Evaluation of a single-channel portable monitor for the diagnosis of obstructive sleep apnea. J Clin Sleep Med 2011;7(4):384-90.

25. Netzer N, Eliasson AH, Netzer C, et al. Overnight pulse oximetry for sleep-disordered breathing in adults: a review. Chest 2001;120(2):625-33.

26. Kapur VK, Auckley DH, Chowdhuri S, et al. Clinical practice guideline for diagnostic testing for adult 
obstructive sleep apnea: an American Academy of sleep medicine clinical practice guideline. J Clin Sleep Med 2017;13(3):479-504.

27. Berry RB, Budhiraja R, Gottlieb DJ, et al. Rules for scoring respiratory events in sleep: update of the 2007 AASM manual for the scoring of sleep and associated events. Deliberations of the sleep apnea definitions task force of the American academy of sleep medicine. J Clin Sleep Med 2012;8(5):597-619.

28. Uddin MB, Chow CM, Su SW. Classification methods to detect sleep apnea in adults based on respiratory and oximetry signals: a systematic review. Physiol Meas 2018;39(3):03TR01.

29. Del Campo F, Crespo A, Cerezo-Hernandez A, et al. Oximetry use in obstructive sleep apnea. Expert Rev Respir Med 2018;12(8):665-81.

30. Mendonca F, Mostafa SS, Ravelo-Garcia AG, et al. A review of obstructive sleep apnea detection approaches. IEEE J Biomed Health Inform 2019; 23(2):825-37.

31. Azarbarzin A, Sands SA, Stone KL, et al. The hypoxic burden of sleep apnoea predicts cardiovascular disease-related mortality: the osteoporotic fractures in men study and the sleep heart health study. Eur Heart J 2019;40(14):1149-57.

32. Mendonca F, Mostafa SS, Ravelo-Garcia AG, et al. Devices for home detection of obstructive sleep apnea: a review. Sleep Med Rev 2018;41:149-60.

33. Terrill PI. A review of approaches for analysing obstructive sleep apnoea-related patterns in pulse oximetry data. Respirology 2020;25(5): 475-85.

34. Deviaene M, Testelmans D, Buyse B, et al. Automatic screening of sleep apnea patients based on the SpO2 signal. IEEE J Biomed Health Inform 2019;23(2):607-17.

35. Burgos A, Goni A, Illarramendi A, et al. Real-time detection of apneas on a PDA. IEEE Trans Inf Technol Biomed 2010;14(4):995-1002.

36. Zhang J, Zhang Q, Wang Y, et al. A real-time autoadjustable smart pillow system for sleep apnea detection and treatment. Proceedings of the 12th International conference on Information processing in sensor networks; Philadelphia, Pennsylvania: Association for Computing Machinery. 2013. p. 179-90.

37. Angius G, Raffo L, editors. A sleep apnoea keeper in a wearable device for continuous detection and screening during daily life. 2008. Piscataway: IEEE - Institute of Electrical and Electronics Engineers; 2008. p. 433-6.

38. Mendonca F, Mostafa SS, Morgado-Dias F, et al. An oximetry based wireless device for sleep apnea detection. Sensors (Basel) 2020;20(3):888.

39. McNicholas WT, Bonsigore MR. Sleep apnoea as an independent risk factor for cardiovascular disease: current evidence, basic mechanisms and research priorities. Eur Respir J 2007;29(1):156-78.
40. Lam JC, Mak JC, Ip MS. Obesity, obstructive sleep apnoea and metabolic syndrome. Respirology 2012; 17(2):223-36.

41. Ebben MR, Krieger AC. Diagnostic accuracy of a mathematical model to predict apnea-hypopnea index using nighttime pulse oximetry. J Biomed Opt 2016;21(3):35006.

42. Jung DW, Hwang SH, Cho JG, et al. Real-time automatic apneic event detection using nocturnal pulse oximetry. IEEE Trans Biomed Eng 2018;65(3):706-12.

43. Marcos JV, Hornero R, Alvarez D, et al. Automated prediction of the apnea-hypopnea index from nocturnal oximetry recordings. IEEE Trans Biomed Eng 2012;59(1):141-9.

44. Tkacova R, McNicholas WT, Javorsky M, et al. Nocturnal intermittent hypoxia predicts prevalent hypertension in the European Sleep Apnoea Database cohort study. Eur Respir J 2014;44(4):931-41.

45. Kent BD, Grote L, Bonsignore MR, et al. Sleep apnoea severity independently predicts glycaemic health in nondiabetic subjects: the ESADA study. Eur Respir J 2014;44(1):130-9.

46. Azarbarzin A, Sands SA, Taranto-Montemurro L, et al. The sleep apnea-specific hypoxic burden predicts Incident heart failure. Chest 2020;158(2):739-50.

47. Aaronson JA, van Bezeij T, van den Aardweg JG, et al. Diagnostic accuracy of nocturnal oximetry for detection of sleep apnea syndrome in stroke rehabilitation. Stroke 2012;43(9):2491-3.

48. Ward NR, Cowie MR, Rosen SD, et al. Utility of overnight pulse oximetry and heart rate variability analysis to screen for sleep-disordered breathing in chronic heart failure. Thorax 2012;67(11):1000-5.

49. Malbois M, Giusti V, Suter M, et al. Oximetry alone versus portable polygraphy for sleep apnea screening before bariatric surgery. Obes Surg 2010;20(3):326-31.

50. Chung F, Liao P, Elsaid H, et al. Oxygen desaturation index from nocturnal oximetry: a sensitive and specific tool to detect sleep-disordered breathing in surgical patients. Anesth Analg 2012;114(5):993-1000.

51. Scott AS, Baltzan MA, Wolkove N. Examination of pulse oximetry tracings to detect obstructive sleep apnea in patients with advanced chronic obstructive pulmonary disease. Can Respir J 2014;21(3):171-5.

52. Bassetti CLA, Randerath W, Vignatelli L, et al. EAN/ ERS/ESO/ESRS statement on the impact of sleep disorders on risk and outcome of stroke. Eur Respir J 2020;55(4):1901104.

53. Andres-Blanco AM, Alvarez D, Crespo A, et al. Assessment of automated analysis of portable oximetry as a screening test for moderate-to-severe sleep apnea in patients with chronic obstructive pulmonary disease. PLoS One 2017;12(11):e0188094.

54. Guilleminault C, Connolly S, Winkle R, et al. Cyclical variation of the heart rate in sleep apnoea syndrome. Mechanisms, and usefulness of $24 \mathrm{~h}$ 
electrocardiography as a screening technique. Lancet 1984;1(8369):126-31.

55. Penzel T, McNames J, Murray A, et al. Systematic comparison of different algorithms for apnoea detection based on electrocardiogram recordings. Med Biol Eng Comput 2002;40(4):402-7.

56. Penzel T. Is heart rate variability the simple solution to diagnose sleep apnoea? Eur Respir J 2003; 22(6):870-1.

57. Heneghan C, de Chazal P, Ryan S, et al. Electrocardiogram recording as a screening tool for sleep disordered breathing. J Clin Sleep Med 2008;4(3): 223-8.

58. Pepin JL, Delavie N, Pin I, et al. Pulse transit time improves detection of sleep respiratory events and microarousals in children. Chest 2005;127(3): 722-30.

59. Bar A, Pillar G, Dvir I, et al. Evaluation of a portable device based on peripheral arterial tone for unattended home sleep studies. Chest 2003;123(3): 695-703.

60. O'Donnell CP, Allan L, Atkinson P, et al. The effect of upper airway obstruction and arousal on peripheral arterial tonometry in obstructive sleep apnea. Am J Respir Crit Care Med 2002;166(7):965-71.

61. Dvir I, Adler Y, Freimark D, et al. Evidence for fractal correlation properties in variations of peripheral arterial tone during REM sleep. Am J Physiol Heart Circ Physiol 2002;283(1):H434-9.

62. Yalamanchali S, Farajian V, Hamilton C, et al. Diagnosis of obstructive sleep apnea by peripheral arterial tonometry: meta-analysis. JAMA Otolaryngol Head Neck Surg 2013;139(12):1343-50.

63. Peppard PE, Young T, Barnet JH, et al. Increased prevalence of sleep-disordered breathing in adults. Am J Epidemiol 2013;177(9):1006-14.

64. Cuspidi C, Tadic M, Sala C, et al. Blood pressure non-dipping and obstructive sleep apnea syndrome: a meta-analysis. J Clin Med 2019;8(9):1367.

65. Genta-Pereira DC, Furlan SF, Omote DQ, et al. Nondipping blood pressure patterns predict obstructive sleep apnea in patients undergoing ambulatory blood pressure monitoring. Hypertension 2018; 72(4):979-85.

66. Crinion SJ, Ryan S, Kleinerova J, et al. Nondipping nocturnal blood pressure predicts sleep apnea in patients with hypertension. J Clin Sleep Med 2019; 15(07):957-63.

67. Marrone O, Bonsignore MR. Blood-pressure variability in patients with obstructive sleep apnea: current perspectives. Nat Sci Sleep 2018;10:229-42.

68. Davies RJ, Jenkins NE, Stradling JR. Effect of measuring ambulatory blood pressure on sleep and on blood pressure during sleep. BMJ 1994; 308(6932):820-3.

69. Davies RJ, Crosby J, Vardi-Visy K, et al. Non-invasive beat to beat arterial blood pressure during
non-REM sleep in obstructive sleep apnoea and snoring. Thorax 1994;49(4):335-9.

70. Lazazzera R, Belhaj Y, Carrault G. A new wearable device for blood pressure estimation using Photoplethysmogram. Sensors (Basel) 2019;19(11):2557.

71. Practice parameters for the use of actigraphy in the clinical assessment of sleep disorders. American Sleep Disorders Association. Sleep 1995; 18(4):285-7.

72. Morgenthaler T, Alessi C, Friedman L, et al. Practice parameters for the use of actigraphy in the assessment of sleep and sleep disorders: an update for 2007. Sleep 2007;30(4):519-29.

73. Sadeh A, Hauri PJ, Kripke DF, et al. The role of actigraphy in the evaluation of sleep disorders. Sleep 1995;18(4):288-302.

74. Sadeh A. The role and validity of actigraphy in sleep medicine: an update. Sleep Med Rev 2011;15(4):259-67.

75. Martin JL, Hakim AD. Wrist actigraphy. Chest 2011; 139(6):1514-27.

76. Goldstone A, Baker FC, de Zambotti M. Actigraphy in the digital health revolution: still asleep? Sleep 2018;41(9).

77. Marino M, Li Y, Rueschman MN, et al. Measuring sleep: accuracy, sensitivity, and specificity of wrist actigraphy compared to polysomnography. Sleep 2013;36(11):1747-55.

78. Salmi T, Telakivi T, Partinen M. Evaluation of automatic analysis of SCSB, airflow and oxygen saturation signals in patients with sleep related apneas. Chest 1989;96(2):255-61.

79. Zaffaroni A, de Chazal P, Heneghan C, et al. SleepMinder: an innovative contact-free device for the estimation of the apnoea-hypopnoea index. Annu Int Conf Proc IEEE Eng Med Biol Soc 2009;2009:7091-4.

80. Zaffaroni A, Kent B, O'Hare E, et al. Assessment of sleep-disordered breathing using a non-contact bio-motion sensor. J Sleep Res 2013;22(2):231-6.

81. Pallin M, O'Hare E, Zaffaroni A, et al. Comparison of a novel non-contact biomotion sensor with wrist actigraphy in estimating sleep quality in patients with obstructive sleep apnoea. J Sleep Res 2014;23(4):475-84.

82. Crinion SJ, Tiron R, Lyon G, et al. Ambulatory detection of sleep apnea using a non-contact biomotion sensor. J Sleep Res 2020;29(1):e12889.

83. Ko PR, Kientz JA, Choe EK, et al. Consumer sleep technologies: a review of the landscape. J Clin Sleep Med 2015;11(12):1455-61.

84. Penzel T, Schöbel C, Fietze I. New technology to assess sleep apnea: wearables, smartphones, and accessories. F1000Res 2018;7:413.

85. Behar J, Roebuck A, Domingos JS, et al. A review of current sleep screening applications for smartphones. Physiol Meas 2013;34(7):R29-46.

86. O'Donnell C, Ryan S, McNicholas WT. The impact of telehealth on the organization of the health system and integrated care. Sleep Med Clin 2020;15(3): 431-40. 